\title{
Thorsten Roelcke
}

\section{Latein als Lingua franca im deutschen Sprachdenken des 17. und 18. Jahrhunderts}

\section{Einleitende Bemerkungen}

Das Lateinische ist in Europa bis zum Beginn der Neuzeit die allgemeine Lingua franca in der internationalen Kommunikation. ${ }^{1}$ Doch erfährt es spätestens seit dem 17. Jahrhundert eine wachsende Konkurrenz durch den Gebrauch von anderen Sprachen wie zum Beispiel dem Italienischen oder dem Französischen. Auch im deutschen Sprachraum ist vom 17. bis zum 19. Jahrhundert eine solche Entwicklung zu beobachten, ${ }^{2}$ die diese Zeit zu einer wichtigen Etappe der deutschen Sprachgeschichte macht. ${ }^{3}$

Angesichts der einsetzenden und zunehmenden Verdrängung wird der Gebrauch des Lateinischen als Lingua franca von zahlreichen Sprachdenkern im Deutschland des Barock und der Aufklärung diskutiert. ${ }^{4}$ Dabei bilden sich insbesondere die folgenden vier Schwerpunkte heraus: die Entstehung und die Entwicklung des Lateinischen als Lingua franca innerhalb gelehrter Kommunikation, die Konkurrenz des Lateinischen zu anderen europäischen Sprachen in Geschichte und Gegenwart, sprachpuristische Forderungen nach der Verwendung der deutschen Sprache anstelle der lateinischen Lingua franca sowie grundsätzliche Erwägungen zur wissenschaftlichen Eignung einzelner Sprachen überhaupt.

Im vorliegenden Beitrag werden diese Schwerpunkte deutscher Sprachreflexion über eine lateinische Lingua franca in der Frühen Neuzeit nachgezeichnet. Die Ergebnisse gehen dabei auf ein Projekt zur Sprachtheorie in Barock und Aufklärung zurück, in welchem etwa 650 Texte ausgewertet wurden, ${ }^{5}$ sowie

1 Vgl. Hattenhauer 1997; Kirkness \& Munske 1996; von Polenz 1994: 347-368; Pörksen 1986; Roelcke 2001; Roelcke 2015a.

2 Vgl. Eisenberg 22012; Gardt 1997; 2001; Härle 1996; Henkel 2004; Jones 1995; Kirkness 1975; Kirkness 1985; Kirkness 1991; 1998; Klein 2011a; 2011b; Löffler 1991; von Polenz 1994: 107-134; Schiewe 1996.

3 Vgl. Eggers 1986; von Polenz 1994; Roelcke 1995: 138-192; Roelcke 1998.

4 Zur Sprachreflexion und zu Sprachkonzeptionen in Barock und Aufklärung vgl. die Standardwerke von Haßler 1984 und Ricken 1989; Ricken 1990.

5 Vgl. insb. Gardt et al. 1991; Quellenverzeichnis in Roelcke 2014a: 354-428.

Ә Open Access. () 2019 Thorsten Roelcke, publiziert von De Gruyter. (cc) BY-NC-ND Dieses Werk ist lizenziert unter der Creative Commons Attribution-NonCommercial-NoDerivatives 4.0 Lizenz.

https://doi.org/10.1515/9783110676631-002 
auf eine Studie zur deutschen Reflexion über die drei alten Sprachen Latein, Griechisch und Hebräisch im 17. und 18. Jahrhundert. ${ }^{6}$

\section{Latein als Lingua franca gelehrter Kommunikation}

Latein findet im deutschen Sprachraum des 17. und 18. Jahrhunderts eine vielfältige Verwendung als Lingua franca der Kommunikation unter gelehrten Personen. Dieser Umstand erfährt bei den Sprachdenkern dieser Zeit recht unterschiedliche Bewertungen. Zwei Beispiele lassen hier das Spektrum von positiver Einschätzung und Anerkennung bis hin zu negativer Beurteilung und Ablehnung deutlich werden: So lobt Johann Klaj das Lateinische als eine „Dolmetscherin der Welt““, 7 während Andreas Rivinus klagt, dass man zu seiner Zeit unter einer „Occidentalischen Lateinischen Monarchie“ ${ }^{\text {8 }}$ lebe.

Ungeachtet solcher Bewertungen besteht über die große Bedeutung des Lateinischen für die gelehrte Kommunikation innerhalb des europäischen Sprachraums weitgehend Einigkeit: Carl Gustav von Hille weist darauf hin, dass das Lateinische im 17. Jahrhundert die internationale Sprache in Europa sei, das Arabische hingegen diejenige in Asien: Man komme „mit der Lateinischen durch gantz Europen und mit der Arabischen durch gantz Asien“. ${ }^{10}$ Johann Joachim Becher zufolge ist eine lateinische Sprachkompetenz eine wesentliche Voraussetzung, um überhaupt an internationaler Kommunikation im Bereich der Gelehrsamkeit teilhaben zu können: Wer „nicht Latein kann / wird nicht vor gelehrt gehalten / dieweil er das Mittel nicht hat gelehrt zu werden“. ${ }^{11}$ Diese prominente Stellung des Lateinischen ist es nach Johann Christoph Adelung letztlich aber auch, welche die Ausbildung einzelner nationaler Wissenschaftssprachen bis in das 17. Jahrhundert behindert habe: Die Ausbildung einer deutschen Sprache der Gelehrsamkeit hatte sich bereits seit geraumer Zeit „von den Universitäten am wenigsten zu versprechen, weil das barbarische Latein alle Lehrstühle beherrschte“. ${ }^{12}$

\footnotetext{
6 Vgl. Roelcke 2014a; zur Reflexion über das Französische Roelcke 2014b.

7 Klaj 1645/1965: 2.

8 Rivinus 1653: 13.

9 Vgl. Roelcke 2015b; Roelcke 2016.

10 Hille 1647/1970: 83f.

11 Becher 1674: 43.

12 Adelung 1782a: 58.
} 
Der Prozess der Herausbildung des Lateinischen zu einer Lingua franca im Allgemeinen und $\mathrm{zu}$ einer internationalen Wissenschaftssprache im Besonderen wird von zahlreichen Sprachdenkern der Zeit erörtert. So entwirft beispielsweise Friedrich Andreas Hallbauer eine durchaus kritische Sicht auf die Geschichte der lateinischen Sprache im deutschen Raum: Nach dem Niedergang des Römischen Reichs sei in Gottesdiensten zunächst aus echter „Hochachtung der lateinischen Sprache“ am Lateinischen festgehalten worden; um aber das „Volk in Unwissenheit zu erhalten“ und aus Streben nach „Macht und Hoheit“ habe man darauf eine ,abergläubische Hochachtung vor der lateinischen Sprache“ entwickelt und „die teutsche Sprache, als eine ungeschickte und unfähige, “ disqualifiziert. ${ }^{13}$ Im Weiteren habe man schließlich ,auch in Gerichten und Canzeleyen die lateinische Sprache eingeführet und diese bey dem Käyser, auch wol grossen Chur-Fürsten, die Erz-Bischöffe und Bischöffe, sonst aber insgemein bey den Fürsten die Aebte, Probste und canonici willig gebrauchen“ lassen; im Rahmen dieses erweiterten Gebrauchs des Lateinischen „blieb nun die teutsche Sprache liegen, und wurde durch fremde Wörter vermischet: ja die alte teutsche Schrift wurde so gar unterdruckt und ausgetilget“ “. ${ }^{14}$

Hallbauer skizziert einen Weg, nach dem das Lateinische zunächst in Kirche und Theologie und später in Bildung und Wissenschaft, Politik und Verwaltung sowie in Rede- und Dichtkunst Verwendung gefunden hat. Eine solche Entwicklung beschreibt auch Johann Christoph Adelung, der das Bildungsmonopol und die Verwaltungsmacht der Geistlichkeit für die Bevorzugung der lateinischen und die Vernachlässigung von anderen Sprachen im Mittelalter verantwortlich macht:

Da die Geistlichen, welche anfänglich gemeiniglich Ausländer waren, aus Bequemlichkeit die Lateinische Sprache nicht allein zur Sprache des Gottesdienstes machten, sondern sie auch, weil sie die einigen Gelehrten dieser Zeit, ja die einigen waren, welche schreiben und lesen konnten, in Schriften und öffentlichen Verhandlungen einführten, weil man glaubte, die neuen Begriffe, welche man durch die Cultur erhielt, ließen sich in der alten barbarischen Volkssprache nicht ausdrucken, so, blieb diese in der Cultur zurück, und daher siehet z. B. die Deutsche Sprache dieser Zeit noch so roh und wild aus, als das Volk, welches derselben überlassen war. ${ }^{15}$

Nicht allein im Mittelalter, sondern auch in der Frühen Neuzeit hat sich das Lateinische nach Auffassung der deutschen Sprachdenker des Barock und der Aufklärung als Lingua franca gegenüber anderen Sprachen Europas behaupten

13 Hallbauer 1725/1974: 34.

14 Ebd.: $34 \mathrm{f}$.

15 Adelung 1782b: 348. 
können. Thieme führt dies vor allem auf den kanonischen Charakter klassischer Werke zurück, die insbesondere im Zeitalter der Renaissance und des Humanismus eine Vorbildfunktion entfalteten und den Gebrauch des Lateins geradezu zu einer Modeerscheinung werden ließen:

Da die Auffindung der römischen und griechischen Schriftsteller überhaupt zur Wiederherstellung der Wissenschaften die nähere Veranlassung ward: so blieben nicht nur diese Schriften vom Anfange lange Zeit die einzige - in der Folge wiederum lange Zeit die vorzüglichste Quelle der ganzen eleganten Gelehrsamkeit; sondern auch die Sprachen, in welchen sie abgefasst waren, wurden als ein Schmuck angesehen, von welchem man jene Wissenschaften selbst, nicht ohne sie zu schänden, entkleiden könnte. Auf diese Art ward besonders die lateinische Sprache das Organ der ganzen Gelehrsamkeit. Man schrieb und sprach, las und lehrte, disputirte und examinirte lateinisch; und Alles, was nur ein wenig gelehrten Anstrich haben wollte, lebte und webte im Latein. ${ }^{16}$

Erst in der zweiten Hälfte des 18. Jahrhunderts kann dann etwa Jakob Hemmer schließlich feststellen, dass sich das Deutsche als Sprache in vielen Bereichen der Gelehrsamkeit hat durchsetzen und dabei den Gebrauch des Lateinischen verdrängen können:

Deutschland hat nun nicht mehr nöthig, in Bearbeitung der Künste und Wissenschaften eine fremde Sprache zu reden, wie es so viele Jahrhunderte gethan hat, da ein grosen Theils steifes und ungeschmackhaftes Latein in seinen Büchern herrschete. Alles, was zur Sprach- Dicht- und Redekunst, was zur Geschichte, zur Rechts- und Gottesgelehrtheit, zur Vernunft- und Naturlehre, zur Grundwissenschaft (Metaphysica), zur ganzen Weltweisheit und Gröselehre (Mathesis) gehöret, alle Theile der menschlichen Kenntnisse lassen sich jetzt auf eine geschickte und vortreffliche Art deutsch einkleiden. ${ }^{17}$

Im Zuge dieser Entwicklung hat die deutsche Sprache einen starken Wandel im Bereich fachsprachlicher Lexik erfahren. Laut Friedrich Gedike zeichnet sie sich in dieser Zeit durch eine große Menge an neuen Wörtern, Wendungen und Wortfügungen aus, „so daß sie [...] izt der Philosophie und Poesie gleich bequem geworden“. ${ }^{18}$ Der Gebrauch des Deutschen anstelle der lateinischen Lingua franca führe dabei inzwischen so weit, dass es „izt dem deutschen Philosophen, der die philosophische Urbarheit seiner Sprache kent, schwer fällt, lateinisch, versteht sich, nicht im scholastischen Mönchslatein, zu schreiben“. ${ }^{19}$

Auch Georg Friedrich Meier vertritt diese Einschätzung, wenn er bereits einige Jahre zuvor schreibt: „Die lateinische Sprache hat beynahe aufgehört, die

16 Thieme 1789: $166 \mathrm{f}$.

17 Hemmer 1775: $4 \mathrm{f}$.

18 Gedike 1779: 389.

19 Ebd. 
Muttersprache der Gelehrten zu seyn, und die Bemühung der meisten Gelehrten scheint dahin zu gehen, statt des Lateins die deutsche Sprache in der gelehrten Welt zur vornehmsten und gewöhnlichsten Sprache $\mathrm{zu}$ machen“. ${ }^{20}$ Doch erkennt Meier in dieser Entwicklung auch die Gefahr einer Provinzialisierung der Kommunikation im Bereich der Gelehrsamkeit und mahnt daher zu Besonnenheit: Es sei zu prüfen, „ob diese Veränderung in dem Zustande der Gelehrsamkeit unter den Deutschen, der Gelehrsamkeit zum Vortheil oder zum Schaden gereiche“. 21

\section{Konkurrenz des Lateinischen mit anderen Sprachen}

In der deutschen Sprachreflexion des Barock und der Aufklärung wird wiederholt die Konkurrenz zwischen der lateinischen Lingua franca und der deutschen Sprache erörtert. Dabei stehen die Bereiche Kirche und Theologie, Bildung und Wissenschaft, Politik und Verwaltung sowie Rede- und Dichtkunst im Vordergrund.

Die große Bedeutung des Lateinischen in Kirche und Theologie zur Zeit des Mittelalters und der Frühen Neuzeit wird dabei meist kritisch bewertet, insbesondere mit Blick auf die Verwendung von Latein im Gottesdienst. Wiederholte Bemühungen, die deutsche Sprache neben der lateinischen als Predigtsprache zu etablieren (wie sie insbesondere für protestantisch geprägte Regionen des deutschen Sprachraums üblich sind), fasst zum Beispiel Friedrich Andreas Hallbauer zusammen:

Nachdem die Teutschen zum Christlichen Glauben gebracht, wurde mit dem Gottesdienste die lateinische Sprache, zu nicht geringen Schaden der Mutter-Sprache, eingeführet: iedoch gab es oft Fälle, da man dieser sich nothwendig mündlich und schriftlich bedienen mußte: daher der Gebrauch derselben nie gäntzlich aufhören können. Uber dieses funden sich auch Verschiedene, welche durch höchstrühmliche verordnungen, auch eigenes Bemühen, der teutschen Sprache aufzuhelfen trachteten: unter welche vornehmlich die Kayser, Carl der Grosse, drey Frideriche, Rudolph, Maximilian, und Carl der fünfte zu rechnen sind.22

20 Meier 1763: 3.

21 Ebd.

22 Hallbauer 1725/1974: 33. 
Einen anschaulichen und durchaus unterhaltsamen Einblick in den Gebrauch des Lateinischen im allgemeinen akademischen Betrieb in der Zeit des Barock gibt (am Beispiel der Universität Leipzig) Christian Weise:

Ferner giebt es auff Universitäten Lateinisch zu reden / wenn man umb ein beneficium anhält. Also müssen an etlichen Orten die Candidati Baccalaureatus und Magisterii durch den ersten auf ihrer Ordnung ihr Desiderium in einer kurtzen Rede vortragen. Also auch unter den Magistris, wer in die Facultät kommen will / der muß seine Disputationes pro Loco, und wie es an etlichen Orten genennet wird / die Inspectionem Schedularum, und endlich die receptionem durch eine Lateinische Rede bey der Facultät erhalten. Ja / wer zu Leipzig eine Collegiatur haben will / von dem wird erfordert / daß er bey einem iedweden Collegiatem in specie umb ein gutes Votum bittet. ${ }^{23}$

„Deutsch reden und schreiben wird in Teutschland“ nach Kaspar Stieler also im ausgehenden 17. Jahrhundert im wissenschaftlichen Bereich noch „vor eine der geringsten Künste geschätzet“. ${ }^{24}$ So würden das „Lateinische und Griechische, die Römische und Attische Sprachen, zum Studium der Philosophie, zu Erlernung mancherley Weysheit“ verwendet, während „das Hebräische, die Morgenländische, zum Studium der Theologie, zu Begreifung der göttlichen Geheimnüße“ sowie „die romanischen und andere Sprachen“ im Allgemeinen „zur Bezier- und Erhebung scharfer Gedanken und Erfindungen“ eingesetzt würden. ${ }^{25}$ Dies ändert sich erst im Verlaufe des 18. Jahrhunderts, indem das Deutsche auch in diesem Bereich zunehmend gebraucht wird und das Lateinische (mit Ausnahme etwa in der Theologie) weitgehend verdrängt.

Es werden jedoch auch Stimmen laut, die noch Ende des 18. Jahrhunderts für eine Verwendung des Lateinischen als internationaler Wissenschaftssprache in der Philosophie werben: „Die lateinische Sprache ist insonderheit diejenige, vermittelst welcher die Gelehrten verschiedner Nazion sich einander mitteilen, und ihre Angelegenheiten untereinander abmachen, - könten und sollten“. ${ }^{26}$ Hierzu bedürfe es jedoch weiterer sprachpflegerischer Bemühungen: Um die lateinische Lingua franca „,bequemer $\mathrm{zu}$ haben, sollte man von allem puristischen Vorurteil absehen, und sich die Freiheit nehmen, mit ihr nach filosofischem Wilkür zu schalten“; auf diese Weise könne „dermaleinst aus der lateinischen eine der Mängel aller andern entledigte, volkommen zwekmäßige filosofische Sprache werden“. ${ }^{27}$

23 Weise 1681/1974: 851.

24 Stieler 1691/1968: o.P.

25 Ebd.

26 Richter 1784: 10.

27 Ebd.: $10 \mathrm{f}$. 
Die lateinische Lingua franca findet im deutschen Sprachraum des 17. und 18. Jahrhunderts nicht nur in den Bereichen Kirche und Theologie sowie Wissenschaft und insbesondere Philosophie, sondern auch in Politik und Verwaltung Gebrauch. Hier steht sie nicht allein in einer Konkurrenz zur deutschen, sondern auch zur französischen und italienischen Sprache: Insbesondere in „StaatsSchrifften, so die Angelegenheiten und Rechte hoher Häupter und Potentzen betreffen, ist es nun dahin gediehen, dass man nicht nur des Lateinischen, sondern auch des Frantzösischen und Welschen sich schwerlich allerdings entbrechen kann“. ${ }^{28}$ Hieraus ergeben sich im Laufe der Zeit durchaus auch Konsequenzen für die mehrsprachliche Kompetenz von Sekretären, die „nebst der Lateinischen und Frantzösischen, insonderheit der Teutschen Feder mächtig seyn" sollten. ${ }^{29}$

Der Grund für den prominenten Gebrauch des Lateinischen in Politik und Verwaltung liegt für viele Sprachdenker des Barock und der Aufklärung in einer Übernahme der römischen Verwaltungssprache im frühen Mittelalter. Dabei wird von einer lateinisch-deutschen Zweisprachigkeit in der öffentlichen Kommunikation seit karolingischer Zeit ausgegangen: „Carolus M. ließ die Jugend so wol in der lateinischen als teutschen Poesie und Beredsamkeit anführen. Es wurde nach dem so gar in den Canzeleyen und Gerichten, auch auf den Reichstägen die Verträge, Lehnbriefe, Reichstagsabschiede etc. in lateinischer Sprache abgefasset“. 30

Diese Mehrsprachigkeit wird nach Hallbauer bis in seine eigene Zeit „so wol in Gesandschaftsreden, als in Hof- und Staatsschreiben sonderlich an auswartige Höfe beibehalten, wiewol man sich mehr des in den barbarischen Zeiten eingeführten Lateins, als der alten reinen Römischen Sprache bedienet““.31 Angesichts dieser lateinisch-deutschen Mehrsprachigkeit sollten jedoch Interferenzen zwischen den beiden Sprachen möglichst ausgeschlossen werden - auch in Richtung des Lateinischen: „Die lateinische Sprache betreffend; so solte man auch in dieser die Reinlichkeit beobachten, wenn man sich solcher im politischen Händeln bedienet“. ${ }^{32}$ Hier zeigt sich die Unterscheidung zwischen klassischem Latein (positiv konnotiert) und Mittellatein scholastischer Prägung (negativ konnotiert), wobei der Versuch unternommen werden solle, das klassische Latein zu erhalten.

28 Leibniz 1697/1717/1908: 351.

29 Glaffey 1747: 8.

30 Hallbauer 1736: $23 \mathrm{f}$.

31 Ebd.: 24.

32 Ebd.: 278. 
Eine Konkurrenz zwischen der lateinischen Lingua franca und der deutschen Sprache wird von den deutschen Sprachdenkern des 17. und 18. Jahrhunderts auch im Bereich literarischer Texte angenommen. Als Maßstab der Beurteilung sprachlicher Kunstwerke gilt dabei bereits für Friedrich Spee nicht der Gebrauch einer bestimmten Einzelsprache, sondern die Art und Weise, wie mit dieser Sprache Texte gestaltet werden. Aus diesem Grunde macht er sich für die Ansicht stark, dass in der deutschen Sprache ebenso gut $\mathrm{zu}$ dichten sei wie in der lateinischen:

Daß aber nicht allein in Lateinischer sprach / sondern auch so gar in der Teutschen man recht gut Poetisch reden vnnd dichten könne / wird man gleich auß diesem Büchlein abnehmen mögen / vnd mercken / daß es nicht an der sprach / sondern vielmehr an den personen / so es einmal auch in der Teutschen sprach wagen dörfften / gemanglet habe. ${ }^{33}$

Ungeachtet solcher Überlegungen stellt der Gebrauch der lateinischen Sprache in den klassischen Schriften für zahlreiche Sprachdenker der Zeit ein Vorbild für die deutsche Dichtung dar: Durch die Nachahmung von deren Stil könne auch eine deutschsprachige Literatur entwickelt und gepflegt werden. So bemerkt etwa Siegmund von Birken mit Blick auf Martin Opitz und andere Zeitgenossen: Sie „schreiben lauter Kern / Geist und Nachdruck / reden viel in wenig Worten und zierlich / und folgen der Lateinischen Poesy“. ${ }^{34}$

Die Sprachdenker des Barock und der Aufklärung propagieren und protegieren eine zunehmende Verwendung des Deutschen gegenüber dem Lateinischen in literarischen Texten. Diese Entwicklung ruft bei einigen Zeitgenossen offensichtlich die Sorge hervor, dass durch einen stärkeren Gebrauch des Deutschen das Lateinische letztlich ganz verdrängt werden könnte. So heißt es etwa bei Georg Philipp Harsdörffer: „Viel stehen in dem Wahn / daß durch Erhebung der Teutschen Sprache die Lateinische fallen werde aller massen man sihet daß auf den hohen Schulen oft mehr Teutsche / als Lateinische Gedichte aufgesetzet werden“.${ }^{35}$ Harsdörffer selbst teilt diese Sorge offensichtlich nicht, sondern plädiert für einen literarischen Gebrauch des Lateinischen als Vorbild für denjenigen des Deutschen: Keine der beiden Sprachen könne unabhängig von der anderen erlernt werden: „Und welche den Griechischen und Lateinischen Poeten die seltene Erfindungen und die meisterzierlichen Handgriffe nicht abmercken / werden ihre Krippelreimen vielmehr zu ihrer Schande als Lob offentlich schautragen“. ${ }^{36}$ Laut Johann Christoph Gottsched dient die Orientierung am

33 Spee 1649/1967: 5.

34 Birken 1679/1973: 174.

35 Harsdörffer 1645/1969: 40.

36 Ebd.: $40 \mathrm{f}$. 
Stil lateinischer Werke dazu, den als Schwulst empfundenen Stil früherer Werke deutscher Literatur zu vermeiden: „Jemehr wir nämlich die Füße und Verse der Alten nachahmen können, destomehr Wohlklang und Harmonie hat unsre Sprache und Verskunst aufzuweisen“. 37

\section{Puristische Kritik am Gebrauch des Lateinischen}

Neben solchen Sprachdenkern, die dem Gebrauch des Lateinischen im deutschen Sprachraum durchaus positiv, wenn auch mehr oder weniger differenziert gegenüberstehen, finden sich im deutschen Sprachraum des 17. und 18. Jahrhunderts aber auch solche, welche die Verwendung des Lateinischen ganz ablehnen selbst wenn sie dessen rhetorische und literarische Vorbildfunktion kaum infrage stellen. Die Gründe, die hierbei für die Bevorzugung des Deutschen angeführt werden, sind zum einen patriotischer und zum anderen pragmatischer Natur.

Die puristische Forderung, die deutsche anstelle der lateinischen Sprache in wissenschaftlichen Texten $\mathrm{zu}$ verwenden, wird im 17. Jahrhundert vor allem von Philipp von Zesen gestellt. So schreibt dieser zum Beispiel: „Ja es were zu wündschen / daß der fleis und die erschrökliche arbeit / die etliche auf die Lateinische sprache gewendet / auf ihre muttersprache / die es würdiger ist / anwendeten / und so wohl ihr / als ihnen einen unsterblichen nahmen machten“. ${ }^{38}$ Eine Kritik am Gebrauch des Lateinischen als wissenschaftlicher Sprache ist aber auch zu Beginn des 18. Jahrhunderts zu finden: Viele Gelehrte „jetziger Zeit“ würden „gäntzlich auf die Lateinisch- oder andere Sprach sich verlegen“ und die deutsche Sprache, „weil sie dann nichts wenigers verstehen (so gelehret sie auch nur seyn mögen oder wollen) als ihre Teutsche Muttersprach“, vernachlässigen oder gar verachten: „Ars non habet hostem nisi ignorantem“. ${ }^{39}$ Friedrich Gottlieb Klopstock unterstreicht in seinem fiktionalen Text der Gelehrtenrepublik noch zum Ende des 18. Jahrhunderts diese Forderung mit einer sprachpatriotischen Haltung und bezieht sich dabei vor allem auf die Bereiche der Theologie und der Philosophie:

Die jetzigen Scholasten, die jenen nun das hundertmal nachsprechen, sind weiter nichts, als lateinische oder griechische Sprachmeister. Wer verachtet sie deswegen, weil sie nur

37 Gottsched 1742/1973: 467.

38 Zesen 1651/1974: 241.

39 Parnassus Boicus 1726: 298. 
das sind? Aber sollen sie denn deswegen, weil sie nur das sind, auch fortfahren eine Zunft zu seyn? Und dennoch würde die unüberwindliche deutsche Geduld sie noch beybehalten; wenn sie den Fortgang der Wissenschaften, durch Verwaltung der Nebendinge in Hauptsachen, des Mittels in den Zweck, nicht hinderten; nicht, weil man Anmerkungen über die Alten gar füglich lateinisch schreibt, noch immer bey ihrem Wahne blieben, daß man überhaupt am besten thäte in dieser Sprache zu schreiben; und, welches vollends alles übertrift, was nur ungedacht und lächerlich ist, daß man in keiner neuern, sondern einzig und allein in der römischen Sprache, (thun sie's etwa? und kann man's jezo noch?) schön schreiben könnte; wenn sie uns endlich, vornämlich durch diese Behauptung, nicht gerade zu verführen wollten, Hochverräther an unserm Vaterlande, an uns selbst, und an unsern Nachkommen zu werden, und zu glauben, die wahre, ihre, tiefeingeprägte Kraft und Schönheit des deutschen Geistes könne durch unsre Sprache nicht ausgedrückt werden. ${ }^{40}$

Eher provokativ schreibt Klopstock an anderer Stelle: „Wer lateinisch schreibt (die bekanten Nothdurften ausgenommen) wird so lange Landes verwiesen, bis er etwas in unsrer Sprache geschrieben hat“. ${ }^{41}$ Doch wird in der Gelehrtenrepublik auch der exklusive Gebrauch einer nationalen Einzelsprache mit Blick auf eine mögliche Provinzialisierung problematisiert: „Wir wissen [...], daß wir uns sondern, und was wir wagen“, denn die wissenschaftliche Landschaft in Europa sei nach wie vor eine ,grosse lateinische Republik“. ${ }^{42}$

Die Beherrschung der lateinischen Sprache gilt bei zahlreichen Gelehrten des 17. und 18. Jahrhunderts als eine Voraussetzung für die erfolgreiche Beschäftigung mit Wissenschaft und Kunst. Doch gibt es laut Christoph Helwig und Joachim Jung durchaus auch Umstände, in denen der Gebrauch anderer Sprachen wie des Deutschen sinnvoller erscheint als der des Lateinischen: „So gibt's ja die erfahrung / das es eben so nötig ist / wo nicht nötiger ein rechtschaffene Teutsche Rede zustellen / als ein Lateinische“. ${ }^{43}$ Neben sprachpatriotischen werden hierfür insbesondere pragmatische Argumente geltend gemacht. So sei etwa das Deutsche dem Lateinischen aus Gründen der Verständlichkeit und Wirtschaftlichkeit vorzuziehen:

Auch seind bißhero alle Sprachen / Künste vnd Wissenschafften an die Lateinische Sprache gebunden / also dz die Lateinische / gleichsam eine Tyrannin vber die andern Sprachen vnd Künste herrschet / der gestalt / daß niemand Hebreisch Griechisch / oder auch weißheit vnd Künste lernen kann / ehe dann er sich in der Lateinischen Sprach wol abgearbeitet / da doch viel füglicher ein jegliche Sprach stracks aus der Muttersprach gelehret / auch alle wissenschaften vnd Künste mit vorteil leichtlich / vnd außführlich in der

40 Klopstock 1774/1975: 118.

41 Ebd.: 24.

42 Ebd.: 129.

43 Helwig \& Jung 1614/1615/1893: 71. 
Deutschen Sprach studiret / vnnd folgends in andern nützlichen Sprachen fast mit einer Mühe mögen getrieben werden. ${ }^{44}$

Dies gelte letztlich sehr weit für „alle Künste vnd Wissenschafften / als Vernunfftkunst / Sitten- vnd Regierkunst / Maß- Wesen- Naturkündigung / ArtzeneyFigur- Gewicht- Stern- Baw- Befestkunst / oder wie sie Nahmen haben mögen““.45

Mit Blick auf die Lebensarbeitszeit eines Menschen lenkt es nach Georg Philipp Harsdörffer von dem eigentlichen Studium der Wissenschaft ab, zunächst Latein zu lernen und sich erst daraufhin mit deren Themen zu beschäftigen. Der Gebrauch des Deutschen anstelle des Lateinischen könne darüber hinaus zu der Ausbildung einer nationalen Literatursprache beitragen, wie sie in anderen europäischen Ländern wie Italien, Frankreich und Spanien bereits bestehe: „Wir Teutsche könten es auch dahin bringen / damit wir so viel Zeit in Erlernung des Lateins nicht verlieren dörfften / sondern so bald mit den zarten Jahren die Wissenschaften selbsten angehen könten““ ${ }^{46}$

Friedrich Andreas Hallbauer betrachtet noch einige Jahrzehnte später das Deutsche zum Gebrauch als Sprache der Gelehrsamkeit als ebenso "geschickt“ wie das Lateinische, doch sei Latein „einem Gelehrten nach den ietzigen Umständen unentbehrlich“, sodass er diejenigen „Studiosi“ kritisiert, die eine "gründliche Erlernung der lateinischen Sprache, als unnöthig“ erachteten. ${ }^{47}$

Ein weiteres Argument, das insbesondere im 18. Jahrhundert gegen den Gebrauch der lateinischen und für denjenigen der deutschen Sprache in Bildung und Wissenschaft vorgebracht wird, zielt darauf ab, dass Latein wiederholt zur Verschleierung mangelhafter fachlicher Kompetenz oder aus akademischer Wichtigtuerei gegenüber sprachlich Unkundigen verwendet werde: „Wenn einige Schulgelehrten nun auch die allergemeinsten Gedanken lateinisch und griechisch ausdrücken, so hoffen sie sich dadurch das Ansehen der Gelehrsamkeit zu geben, weil es ihrer Meinung nach gelehrt klingt“; ${ }^{48}$ solche Personen „,riechen [...] immer nach der Schule, und bilden sich ein, man würde sie für keine Gelehrte halten, wenn sie nicht beständig lateinisch oder griechisch von sich hören lassen“. 49

Bei allen Unterschieden im Detail kommen doch zahlreiche Sprachdenker des Barock und der Aufklärung letztlich darin überein, dass ein vollständiger

44 Ebd.: 66.

45 Ebd.: $71 \mathrm{f}$.

46 Harsdörffer 1645/1969: 24.

47 Hallbauer 1725/1974: $41 \mathrm{f}$.

48 Meier 1763: 76.

49 Ebd. 
Verzicht auf den Gebrauch der lateinischen Lingua franca im akademischen Kontext kaum sinnvoll und wünschenswert sei. Hier erscheint wiederum eine deutsch-lateinische Zweisprachigkeit als Ziel, so zum Beispiel auch bei Christian Thomasius, der eine solche Zweisprachigkeit innerhalb der Jurisprudenz als vorteilhaft erachtet: Ein „Studiosus Juris“ solle einerseits „die lateinische / als eine todte Sprach / nicht gantz und gar verachte[n]: weil sie doch auf gewisse Art die Sprache der Gelehrten ist“; andererseits solle er nicht denken, „sie sey eintzig und allein zu der Weißheit nöthig / und es sey unbillig in andern Sprachen gelehrte Sachen vorzutragen“..$^{50}$

Mit Blick auf fachliche Lexik spricht sich auch Carl Gotthelf Müller für den Gebrauch des Deutschen als Wissenschaftssprache aus, ohne jedoch die Bedeutung der lateinischen Sprache hinsichtlich ihrer Termini in Abrede zu stellen:

Die Sprache Latiens hatte sonsten das Recht, die allgemeine Sprache der Gelehrten zu seyn, behauptet; und wir wollen ihr auch diesen Vorzug, wegen der eingeführten Kunstwörter, einigermaßen nicht streitig machen; indessen ist es doch nicht unmöglich, wenn die teutschen Gelehrten eben diese Ehre ihrer eigenen Sprache bey ihren Landesleuten zuwege zu bringen suchten, daß solche auch zum Vortrage der wichtigsten Wahrheiten aus den höhern Wissenschaften geschickt erfunden werde. ${ }^{51}$

Einen Gebrauch des Lateinischen und des Deutschen fordert etwa auch Georg Litzel: „Wer unter den Weisen weise ist, der bindet sich nicht völlig an die lateinische, sondern auch an die Muttersprache, als welcher er verwandt und von Natur gebunden ist“".52 Als Begründung hierfür gibt Litzel den Sprachgebrauch der römischen Gelehrten selbst an, die sich ihrerseits nicht der griechischen oder ägyptischen, sondern eben der lateinischen Sprache bedient hätten: „Die alten Weisen der Egyptier, Griechen und Römer erkanten solches wohl, und hielten es für eine Thorheit, in einer fremden Sprache zu philosophiren “. ${ }^{53}$

Über Patriotismus und Pragmatismus hinaus ist auch die Vorstellung von Bildung für weite Kreise der Bevölkerung für die Sprachdenker der Aufklärung, aber auch des Barock ein nicht zu unterschätzendes Argument dafür, neben dem Gebrauch der lateinischen Lingua franca auch den der deutschen Sprache zu propagieren. So fordert bereits zu Beginn des 17. Jahrhunderts Johann Amos Comenius, Wissenschaft und Kunst nicht allein in lateinischer, sondern auch in deutscher Sprache zu lehren, um so breiten Bevölkerungskreisen den Zugang zu

50 Thomasius 1713: 141.

51 Müller 1754: 13.

52 Litzel 1730: 85.

53 Ebd.: $85 \mathrm{f}$. 
Bildung zu ermöglichen. Er zeigt sich somit als recht früher Vertreter eines auf so etwas wie Volksaufklärung ausgerichteten Gedankenguts:

Wir wünschen daher inständig, die Studien der Weisheit mögen nicht mehr nur in lateinischen Schriften gelehrt werden, so dass sie in den Kerkern der Schulen festgehalten werden. So ist es bisher in tiefster Verachtung des Volkes und der Volkssprachen zu Unrecht geschehen. Vielmehr soll jedem Volk alles in der eigenen Sprache gelehrt und dadurch allen, die Menschen sind, die Möglichkeit geboten werden, sich lieber mit diesen freien Künsten zu befassen als sich mit den Sorgen dieses Lebens oder ehrgeizigen Bestrebungen, Zechgelagen und allen übrigen Nichtigkeiten abzumühen, wie es beständig geschieht, und so gleichzeitig ihr Leben und ihre Seele zugrunde richten. ${ }^{54}$

Neben der Bildung breiter Bevölkerungsanteile verspricht sich Comenius hiervon auch einen positiven Beitrag zur Entwicklung einer deutschen Literatursprache selbst. Er legt daher einige seiner Werke sowohl in lateinischer als auch in deutscher Sprache vor, so zum Beispiel auch Prodomus Pansophiae: „Wir haben daher beschlossen, auch dieses unser Werk, wenn Gott seine Zustimmung gibt, in der lateinischen und in der heimischen Sprache zu veröffentlichen“. 55

In der Didactica Magna begründet Comenius die Zweisprachigkeit bzw. Übersetzung des Werkes aus dem Deutschen ins Lateinische darüber hinaus mit der Hoffnung, sowohl national als auch international breit rezipiert zu werden. Er ist somit als ein früher Vertreter einer Auffassung anzusehen, der zufolge eine nationale und eine internationale Wissenschaftssprache im Hinblick auf verschiedene Rezipientengruppen in Koexistenz stehen sollten: Das Werk „wurde zunächst zum Gebrauch meiner Landsleute in meiner Muttersprache verfaßt, nun aber auf den Rat etlicher achtenswerter Männer ins Lateinische übersetzt, damit sie, wenn möglich, der Allgemeinheit von Nutzen sei“. ${ }^{56}$

Der Geist der Volksaufklärung wird schließlich bei Gottfried Wilhelm Leibniz offenkundig. Leibniz kritisiert die Auffassung einiger Gelehrter, dass „Weisheit nicht anders als in Latein und Griechisch sich kleiden lasse“, und deren Furcht, „es würde der welt ihre mit großen worthen geraffte geheime unwissenheit entdecket werden““. ${ }^{57}$ Im Weiteren fordert er den Gebrauch des Deutschen als Wissenschaftssprache: Der Gebrauch des Lateinischen bremse zum einen die Weiterentwicklung der deutschen Gelehrsamkeit, indem er von den eigentlichen Inhalten ablenke, und behindere zum anderen die Aufklärung der Bevölkerung, indem durch das Lateinische Wissen und Bildung breiten Teilen der Bevölkerung verschlossen blieben:

54 Comenius 1637/1963: 165.

55 Ebd.

56 Comenius 1657/1957: $13 \mathrm{f}$.

57 Leibniz 1682/1846/1907: 302. 
In Teutschland aber hat man annoch dem latein und der kunst zuviel, der Muttersprach aber und der natur zu wenig zugeschrieben, welches denn sowohl bey den gelehrten als bey der Nation selbst eine schädiche würckung gehabt. Denn die gelehrten, indem sie fast nur gelehrten schreiben, sich offt zu sehr in unbrauchbaren dingen aufhalten; bey der ganzen nation aber ist geschehen, daß diejenigen, so kein latein gelernet, von der wißenschaft gleichsam ausgeschloßen worden, also bey uns ein gewißer geist und scharffsinnige gedancken, ein reiffes urtheil, eine zarthe empfindlichkeit deßen so wohl oder übel gefaßet, noch nicht unter den Leuten so gemein worden, als wohl bey den auslandern zu spüren, deren wohl ausgeübte Mutter-sprach wie ein rein polirtes glas gleichsam die scharffsichtigkeit des gemüths befordert und dem Verstand ein durchleuchtende clarheit giebt. ${ }^{58}$

Während Leibniz noch die meisten seiner Werke in lateinischer oder in französischer Sprache erscheinen lässt, legt sein Schüler Christian Wolff bereits zahlreiche Veröffentlichungen in deutscher Sprache vor. Als Grund hierfür gibt er insbesondere an, auch solche Personen erreichen zu wollen, die des Lateinischen nicht mächtig seien und nicht studiert hätten. Die übliche Vorgehensweise, lateinisch zu publizieren, sei alleine keine hinreichende Begründung dafür, dies auch weiterhin zu tun: „Der gemeine Gebrauch entschuldiget nicht: eine Gewohnheit muß vernünfftig seyn und einen guten Grund vor sich haben, wenn man sich darnach achten soll“". ${ }^{59}$ Der Gebrauch des Deutschen verfolge den „Zweck, den ich mir vorgesetzet hatte, daß auch andere meine Schrifften lesen solten, die nicht studiret und niemahls lateinisch gelernet haben““ ${ }^{60}$

Einen weiteren und dabei nicht unbedeutenden Grund für die Wahl des Deutschen als Sprache wissenschaftlicher Veröffentlichungen bildet für Wolff ein mangelndes Vermögen oder eine mangelnde Lust seiner Schüler, sich in lateinischer Sprache mit wissenschaftlichen Inhalten auseinanderzusetzen: „Hingegen werden durch die lateinischen Kunst-Wörter andere abgeschreckt die Bücher zu lesen und sich daraus zu erbauen, die mit dem Latein entweder nicht können, oder nicht mögen zu thun haben““ ${ }^{61}$

\section{Wissenschaftliche Eignung des Lateinischen}

Die Forderung, neben oder anstelle der lateinischen Lingua franca die deutsche Sprache zu verwenden, wird von einigen Sprachdenkern des Barock und der

58 Ebd.: 302f.

59 Wolff 1733/1973: 26.

60 Ebd.: $26 \mathrm{f}$.

61 Ebd.: 27. 
Aufklärung mit dem Hinweis gestützt, dass sämtliche Einzelsprachen im gleichen Maße dazu geeignet seien, wissenschaftliches Gedankengut zum Ausdruck zu bringen. ${ }^{62}$ So heißt es beispielsweise bei Hille: „Es ist zwar die Vernunft an keine gewisse Sprache gebunden: alle Zungen können verständige Gedanken ausreden / und were diesen zu nahe gesagt / daß man nur in Latein / Griechisch oder Hebräisch weiß / in Teutsch aber närrisch seyn sollte“ ${ }^{63}$

Eine solche wissenschaftliche Äquivalenz einzelner Sprachen gelte insbesondere auch für deren Wortschatz: Vor diesem Hintergrund stellt Justus Georg Schottelius zur Diskussion, ob ,teutsche Kunstwörter nicht können eben so gründlich / vernemlich und wollautend ausdrükken und anzeigen das Ding / dessen Kunstmässige Wörter sie sind / [ . . ] als die Griechische oder Lateinische [Sprache]“. ${ }^{64}$ Für Georg Philipp Harsdörffer ist es daher nicht nachvollziehbar, dass man allein , auf Griechisch / Lateinisch / Welsch oder Frantzösisch verständig“ sein und sich gelehrt ausdrücken könne; denn dem Deutschen „ermangelt nicht ein Wort alles und jedes was man nur durchdenken kann / wolverständig auszureden“. 65

Georg Friedrich Meier erklärt das Lateinische zu einer „gelehrten und philosophischen Sprache“.66 Im Unterschied zu solchen Sprachdenkern, die europäische „Hauptsprachen“ anhand von deren kultureller Bedeutsamkeit ausmachen, ${ }^{67}$ möchte Meier jedoch diese Charakterisierung allein anhand einer Evaluation einzelsprachlicher Merkmale vornehmen. Es sei „lächerlich“, bei dem Ausdruck „gelehrte Sprache“ nur ,an die lateinische und griechische Sprache“ zu denken, da nicht allein derjenige „gelehrt heissen könnte, wer nicht eine von diesen beyden Sprachen, oder beyde zugleich, in seiner Gewalt hat“; vielmehr müsse zunächst einmal untersucht werden, ob diese beiden klassischen Sprachen überhaupt ,alle diejenigen Vollkommenheiten in dem gehörigen Grade besitzen, um derenwillen eine Sprache, den Namen einer gelehrten und philosophischen Sprache, verdient“. 68

Meier fordert daher im Weiteren eine Prüfung von strukturellen Merkmalen einzelner Sprachen im Hinblick auf deren Tauglichkeit im wissenschaftlichen Bereich:

62 Zur Diskussion um die wissenschaftliche Eignung des Deutschen und Lateinischen vgl. auch Roelcke 2018.

63 Hille 1647/1970: 136f.

64 Schottelius 1651: 12.

65 Harsdörffer 1645/1969: 27.

66 Meier 1763: 36.

67 So z. B. Becher 1674.

68 Meier 1763: $36 \mathrm{f}$. 
Wer den ganzen Streit, über die Nothwendigkeit der griechischen und lateinischen Sprache zur wahren Gelehrsamkeit vernünftig entscheiden will, der muß einen deutlichen und vollkommenen Begrif von den mannigfaltigen Vollkommenheiten einer gelehrten Sprache haben, und alsdenn untersuchen, ob diese genannten Sprachen nicht nur diese Vollkommenheiten haben, sondern ob auch andere Sprachen mit eben diesen Vollkommenheiten ausgeschmückt sind, oder ob die es nicht sind. ${ }^{69}$

Dessen ungeachtet gesteht jedoch auch Meier ein, dass es diverse wissenschaftliche Disziplinen gebe, die nicht ohne hinreichende Kompetenzen im Lateinischen sinnvoll studiert werden können - so etwa „das römische Recht sowie die griechische und römische Historie“ ${ }^{70}$

Die deutschen Sprachdenker des 17. und 18. Jahrhunderts äußern sich nur selten über das Verhältnis von Latein als europäischer Lingua franca zu anderen europäischen Sprachen neben dem Deutschen. Daher erscheint der Hinweis von Johann David Michaelis bemerkenswert, dass die romanischen Sprachen aufgrund ihrer einzelsprachlichen Besonderheiten nur wenig zum Gebrauch als (internationale) Wissenschaftssprachen geeignet erscheinen, „denn ein Volck versteht das barbarische Latein des andern nicht, weil ein jedes seine eigene Sprache damit vermischet““ ${ }^{71}$ Michaelis leitet daraus die Forderung ab, im internationalen Sprachgebrauch an der lateinischen Sprache festzuhalten: „Dies ist eben die Haupt-Ursache, welche die Gelehrten bewegen soll, sich der reinen und alten Latinität zu befleißigen“. 72

Vor diesem Hintergrund erfahren dann das Lateinische und das Deutsche, die als wissenschaftssprachlich äquivalent angesehen werden, eine genauere Untersuchung. Und so beschäftigt sich Leibniz mit dem Verhältnis von Konkreta und Abstrakta in beiden Sprachen. Er kommt dabei zu der Einschätzung, dass der Wortschatz des Lateinischen mehr Abstrakta und derjenige des Deutschen mehr Konkreta aufweise. Daher eigne sich das Lateinische eher als Fachsprache theoretischer, das Deutsche dagegen als Fachsprache diverser angewandter Wissenschaften und technischer Disziplinen:

Ich finde, dass die Teutschen ihre Sprache bereits hoch gebracht in allen dem, so mit den fünff Sinnen zu begreiffen, und auch dem gemeinen Mann fürkommet; absonderlich in leiblichen Dingen, auch Kunst- und Handwercks-Sachen, weil nemlichen die Gelehrten fast allein mit dem Latein beschäftiget gewesen und die Mutter-Sprache dem gemeinen Lauff überlassen, welche nichts desto weniger auch von den so genandten Ungelehrten nach Lehre der Natur gar wohl getrieben worden. Und halt ich dafür, dass keine Sprache in der

69 Ebd.: $37 \mathrm{f}$.

70 Ebd.: $79 \mathrm{f}$.

71 Michaelis 1760: 74.

72 Ebd. 
Welt sey, die (zum Exempel) von Ertz und Bergwercken reicher und nachdrücklicher rede als die Teutsche. Dergleichen kann man von allen andern gemeinen Lebens-Arten und Professionen sagen, als von Jagt- und Wäid-Werck, von der Schiffahrt und dergleichen. Wie dann alle die Europäer so auffm grossen Welt-Meer fahren, die Nahmen der Winde und viel andere Seeworte von den Teutschen, nehmlich von den Sachsen, Normannen, Osterlingen und Niedrländern entlehnet. ${ }^{73}$

Johann Jakob Bodmer und Johann Jakob Breitinger weisen ebenfalls auf einige lexikalische Unzulänglichkeiten des Deutschen im wissenschaftlichen Bereich hin und führen diese darauf zurück, dass das Lateinische im deutschsprachigen Raum eher als das Deutsche in wissenschaftlichem Zusammenhang Verwendung finde: „Daß uns in der Deutschen Sprache noch viele Begriffe ausbleiben / die keine eigene Nahmen haben / geschicht aus keiner andern Ursache / als weil Deutschlands sinnreichste Köpffe bisher lieber in der Lateinischen als in ihrer Mutter-Sprache geschrieben haben “. ${ }^{74}$ Als Lösung des Problems empfehlen die beiden Schweizer nach dem Vorbild Frankreichs Entlehnungen aus dem Lateinischen und Griechischen: „Die Frantzosen haben sich dieser Erlaubniß neue Wörter zumachen allezeit bedienet / und so wol aus der Deutschen und Griechischen / als sonderbar aus der Lateinischen die schönsten Wörter angenommen “. ${ }^{75}$

Lexikalische Unterschiede zwischen dem Lateinischen und Deutschen stellen für Leibniz letztlich ein gutes Kriterium dar, wissenschaftliche Thesen und Argumente, die in lateinischer Sprache verfasst sind, zu überprüfen: Sind diese auf Deutsch gut wiederzugeben, seien sie ernst zu nehmen; ist dies dagegen nicht der Fall, seien sie als scholastisch zu verwerfen. Eine solche Sprachprüfung lateinischer Texte sei anhand romanischer Sprachen weitaus weniger sinnvoll, da diese mit dem Lateinischen näher verwandt seien und somit inhaltliche Defizite lateinischer Texte sprachlich eher wieder- und weitergäben:

Es hat die deutsche Sprache darin einen trefflichen Vorzug vor der lateinischen und vor denen, die aus der lateinischen entsprossen, daß sie gleichsam ein Probierstein ist rechtschaffener guter Gedanken. Denn den Franzosen, Italienern und Engländern, weil sie die Freiheit haben, lateinische Worte ihres Gefallens einzumischen, ist es leicht, alle Schulgrillen und undienlichen Phantasien der Philosophen in ihrer Sprache zu geben. Hingegen, weil die deutsche Sprache dessen ungewohnt, daher kommt es, daß die Gedanken, die man mit gutem, reinen Deutsch geben kann, auch gründlich sind, was aber sich nicht

73 Leibniz 1697/1717/1908: 330.

74 Bodmer \& Breitinger 1721: 4.

75 Ebd. 
in gutem Deutsch geben läßt, besteht gemeiniglich in leeren Worten und gehört zu der Scholastik. ${ }^{76}$

Die Grammatik der lateinischen Sprache erfährt in Bezug auf ihre Eignung im Rahmen wissenschaftlicher Kommunikation unterschiedliche Beurteilungen. Johann David Michaelis weist auf die geringe Kompositionsneigung im Lateinischen hin. Aus diesem Grunde sei es weniger zur wissenschaftlichen Kommunikation geeignet als etwa das Griechische (oder auch das Deutsche), selbst wenn es sich inzwischen aus historischen Gründen als internationale Wissenschaftssprache zu etablieren vermocht habe:

Es wäre weit glücklicher, wenn das Loos die Griechische Sprache getroffen hätte, bey der doch die Lateinische in der Medicin und Natur borgen muß, und dabey allen dunckel ist, die kein Griechisch verstehen. Die Biegsamkeit des Griechischen in viel tausend neue Zusammensetzungen der Wörter, würde ein Vortheil gewesen seyn, den man anderwärts vergeblich suchet. Große Gelehrten haben es erkannt: allein es ist zu spät, das Loos ist geworfen, ehe man im Stande war zu wählen, und wir sind gezwungen seiner Entscheidung zu folgen. ${ }^{77}$

Johann Gottfried Herder diskutiert ein syntaktisches Merkmal, das bei dem Gebrauch einer Wissenschaftssprache von großer Bedeutung sei, nämlich das Ausbleiben von Inversionen: „Betrachtet eine Philosophische Sprache: wäre sie von einem Philosophen erdacht: so hübe sie alle Inversionen auf“. ${ }^{78}$ Da lateinische Texte (aufgrund ihrer relativ freien Wortstellung) nur wenige Inversionen aufweisen, zeige die lateinische Sprache eine große Eignung, als wissenschaftliche Sprache Verwendung zu finden: „Nehmt die, die am meisten zur Weltweisheit gebraucht wird, die Lateinische, nehmt sie, wie sie in den Büchern der Weltweisheit ist, wenn sie Lehrsäzze und trockene Beweise vorträgt: wie ist sie? Ohne Inversionen meistentheils“. ${ }^{79}$

Die Unterschiede zwischen der lateinischen und der deutschen Grammatik werden bereits von den deutschen Sprachdenkern des Barock und der Aufklärung als ein wesentlicher Grund dafür angesehen, dass die grammatische Terminologie des Lateinischen nicht als geeignet erscheint, die Grammatik der deutschen Sprache zu beschreiben. In der Gelehrtenrepublik weist Klopstock daher darauf hin, dass eine grammatische Betrachtung der deutschen Sprache nicht allein mit der üblichen lateinischen Terminologie auskomme, da zum einen die lateinische Grammatikschreibung selbst noch nicht vollständig sei und zum anderen die

76 Leibniz 1697/1916: 58.

77 Michaelis 1760: 74.

78 Herder 1877: 191.

79 Ebd. 
Grammatik der deutschen Sprache nicht in vollem Umfange derjenigen der lateinischen Sprache entspreche: Es sei klar, „daß eine deutsche Grammatik, in welcher die fremden Kunstwörter gebraucht würden, dennoch nicht ganz ohne deutsche seyn könnte“. ${ }^{80}$ Klopstock gibt hierfür zwei Gründe an. Zum einen „haben diese alten Grammatiker verschiedenes nicht untersucht, was sie hätten untersuchen sollten; man müste also noch einige Kunstwörter mehr haben, als man bey ihnen antrift“; zum anderen „erfodert das Eigentümliche unserer Sprache einige, die in den lateinischen Grammatiken nicht vorkommen konten“. ${ }^{81}$

Ein weiterer Grund für die Verwendung einer deutschsprachigen Terminologie im Rahmen einer Beschreibung der Grammatik der deutschen Sprache besteht laut Klopstock darin, dass die lateinische Terminologie bereits aus der lateinischen Grammatikschreibung bekannt sei und somit die Einschätzung grammatischer Erscheinungen im Deutschen präge, während deutschsprachige Termini im Rahmen einer vom Lateinischen unabhängigen Grammatikschreibung einen vorbehaltlosen Zugang zur deutschen Grammatik erlaubten:

Diejenigen, denen die fremden Kunstwörter durch lange Angewöhnung geläufig sind, können von dieser Sache nicht unpartheyisch urtheilen, wenn sie sich nicht an die Stelle derer setzen, welche diese Kunstwörter nun erst in spätern Jahren, und ohne die geringste Kentnis des Lateins, viel mühsamer lernen müsten, als sie dieselben in früheren, mit dem Lateine zugleich, gelernt haben. ${ }^{82}$

\section{Abschließende Bemerkungen}

Die deutsche Sprachreflexion des Barock und der Aufklärung setzt sich intensiv mit der lateinischen Lingua franca und deren Gebrauch im deutschen Sprachraum auseinander. Dabei sind insbesondere vier Schwerpunkte auszumachen, die hier noch einmal kurz zusammengefasst seien:

1. Die faktische Bedeutung des Lateinischen als internationaler Lingua franca, die auch im deutschen Sprachraum Verwendung findet, wird von nahezu allen Sprachdenkern des Barock und der Aufklärung anerkannt. Im Hinblick auf ihre Geschichte wird insbesondere auf ihre Herausbildung in Theologie und Kirche im Mittelalter hingewiesen; von hier sei das Lateinische in zahlreiche andere Bereiche wie Bildung und Wissenschaft, Politik

80 Klopstock 1774/1975: 123.

81 Ebd.: $123 \mathrm{f}$.

82 Ebd. 
und Verwaltung sowie Rede- und Dichtkunst vorgedrungen. Dabei erscheint die zweite Hälfte des 18. Jahrhunderts als wichtige Phase in der Umstellung von einem lateinischen auf einen deutschen Sprachgebrauch.

2. Zahlreiche Sprachdenker der Zeit setzen sich vor diesem Hintergrund mit der Konkurrenz zwischen der lateinischen Lingua franca und anderen Sprachen wie etwa dem Deutschen auseinander. Mit Blick auf den Gebrauch in Theologie und Kirche wird dabei auf Versuche hingewiesen, Deutsch als Predigtsprache zu etablieren. Die Verdrängung des Lateinischen durch das Deutsche im akademischen Bereich wird durchaus differenziert betrachtet: Einer positiven Einstellung gegenüber dem klassischen Latein, das es in der Kommunikation verschiedener Bereiche durchaus $\mathrm{zu}$ erhalten gelte, steht eine negative gegenüber dem Mittellatein scholastischer Prägung gegenüber. Vor diesem Hintergrund findet sich wiederholt die Forderung nach einer Zweisprachigkeit in Bezug auf das (klassische) Latein und das Deutsche, unter der die deutsche Sprache dann als eine nationale Literatursprache weiter entwickelt werden könne.

3. Die puristische Kritik daran, ganz oder auch nur zum Teil an der Verwendung der lateinischen Lingua franca neben dem Gebrauch der deutschen Sprache festzuhalten, wird unter den Sprachdenkern des 17. und 18. Jahrhunderts durch zahlreiche Argumente gestützt. Hierzu zählt zum einen ein sprachlich-kultureller Patriotismus, der teils durch den Hinweis auf die Gefahr eines sprachlich-kulturellen Provinzialismus relativiert wird. Zum anderen finden sich zahlreiche pragmatische Hinweise, die für den Gebrauch des Deutschen anstelle des oder (im Falle differenzierter Auseinandersetzung) neben dem Lateinischen angeführt werden: höhere Verständlichkeit und Wirtschaftlichkeit (Nutzung von Lebensarbeitszeit), Vermeidung akademischer Verschleierung und Profilierungssucht, mangelnde Kompetenz und Motivation unter Studenten, der Wunsch, national wie international rezipiert zu werden, und nicht zuletzt die Forderung nach einer Bildung für breitere Bevölkerungskreise (Idee der Volksaufklärung).

4. Unter der Annahme einer strukturellen Gleichwertigkeit einzelner Sprachen wird ein Vergleich der Eignung der lateinischen und der deutschen Sprache für wissenschaftliche Kommunikation angeregt; differenzierte Argumentationen weisen auf bestimmte Bereiche hin, in denen das Lateinische nicht durch andere Sprachen zu ersetzen sei. Der Vergleich selbst bezieht sich dann vor allem auf Abstrakta und Konkreta im wissenschaftlichen Wortschatz; hinsichtlich der Grammatik wird insbesondere auf die reiche Komposition im Deutschen und die Armut an Inversionen im Lateinischen hingewiesen. Wegen seiner relativ großen strukturellen Ferne zum Lateinischen sei das Deutsche besser dazu geeignet, wissenschaftliche Aussagen 
zu überprüfen, als etwa romanische Sprachen. Ein besonderes Augenmerk erfährt die Beschreibung der deutschen Grammatik: Hierfür sei die lateinische Terminologie nicht geeignet, da lateinische Grammatiker einige Erscheinungen des Deutschen nur unvollständig und andere gar nicht erfasst hätten und zudem eine konzeptionelle Unabhängigkeit von der Tradition lateinischer Grammatik vonnöten sei.

Diese und weitere Befunde aus dem Sprachdenken im Deutschland des Barock und der Aufklärung bedürfen (neben der Erhebung von ergänzenden Einzelheiten) der weiteren Analyse und Interpretation. Hierbei ist zum einen an die Diskussion von Parallelen zwischen dem Bestehen einer nationalen Einzelsprache und einer internationalen Lingua franca im 17. und 18. Jahrhundert einerseits sowie im 20. und 21. Jahrhundert andererseits zu denken: Eine solche Diskussion kann dazu beitragen, sowohl Geschichte als auch Gegenwart besser zu begreifen. Zum anderen ist an eine explizite Rückbindung der Befunde an die Sozial- und Kulturgeschichte zu denken: Diese kann helfen, die hier vorgebrachten Thesen und Argumente besser einzuschätzen; umgekehrt sind aber auch vertiefte sozial- und kulturgeschichtliche Erkenntnisse aus einer näheren Betrachtung der Sprachreflexion des 17. und 18. Jahrhunderts zu erwarten.

\section{Literatur}

Adelung, Johann Christoph (1782a): Umständliches | Lehrgebäude | der | Deutschen Sprache, | zur | Erläuterung | der | Deutschen Sprachlehre | für Schulen. | Von | Joh. Christoph Adelung. | Erster Band. Leipzig: Breitkopf.

Adelung, Johann Christoph (1782b): Versuch | einer | Geschichte | der Cultur | des menschlichen Geschlechts. | Von dem Verfasser | des Begriffs menschlicher Fertigkeiten und | Kenntnisse. Leipzig: Hertel.

Becher, Johann Joachim (1674): JOANNIS JOACHIMI BECHERI | Spirensis, | Sacra Casarea Majestatis Consilia|rii, Mathematici, \& Medicina Doctoris, Se|renissimi Bavaria Electoris Medici, olim Eminen|tissimi Electoris Moguntini Persona Medici, \& in | almä Universitate Moguntinä Institutionum | Medicarum Professoris Publici, | \& ordinarii, | METHODVS | DIDACTICA | Seu | CLAVIS ET PRAXIS | SUPER NOVUM SUUM | ORGANON | PHILOLOGICVM, | Das ist: | Gründlicher Beweis / daß die | Weg und Mittel / welche die Schulen biß|hero ins gemein gebraucht / die Jugend zu Erler|nung der Sprachen / insonderheit der Lateinischen / zu füh|ren / nicht gewiß / noch sicher seyen / sondern den Regulen | und Natur der rechten Lehr / und Lern=Kunst schnurstracks | entgegen lauffen / derentwegen nicht allein langweilig / son/dern auch gemeiniglich unfruchtbar / und vergeb|lich ablauffen: | Samt Anleitung zu einem besseren. | Zweyte Edition. Frankfurt: Zunner. 
Birken, Siegmund von (1679/1973): Teutsche | Rede-bind und Dicht-|Kunst / | oder | Kurze Anweisung zur | Teutschen Poesy / | mit Geistlichen Exempeln: | verfasset | durch | Ein Mitglied der höchstlöblichen Frucht|bringenden Gesellschaft | Den Erwachsenen. | Samt dem Schauspiel Psyche | und | Einem Hirten-Gedichte. Nürnberg: Riegel. Nachdruck Hildesheim, New York: Olms.

Bodmer, Johann Jakob \& Johann Jakob Breitinger (1721): Die Discourse der Mahlern. Erster Theil. Zürich: Joseph Lindinner.

Comenius, Johann Amos (1637/1963): Prodomus Pansophiae. In: Vorspiele. Prodomus Pansophiae. Vorläufer der Pansophie. Hrsg., übers., erl. und mit einem Nachwort versehen von Herbert Hornstein. Düsseldorf: Schwann.

Comenius, Johann Amos (1657/1957): DIDACTICA MAGNA | UNIVERSALE OMNES OMNIA | docendi artificium exhibens: | SIVE | Certus \& exquisitus modus, per omnes alicujus Christiani Regni | communitates, Oppida \& Vicos, tales erigendi Scholas, ut Omnis | utriusque sexus Juventus, nemine usquam neglectu, Literis infor|mari, Moribus explori, Pietate imbui, easque ratione intra | pubertatis annos ad omnia qua praesentis \& futura | Vita sunt, instrui possit, | Compendiose, Jucunde, Solide. | Ubi omnium qua svadentur, | FUNDAMENTA, ex ipsissims Rerum natura eruuntur: | VERITAS, artium Mechanicarum parallelis exemplis demonstratur: | SERIES, per Annos, Menses, Dies, Horas, disponitur: | VIA deniqve in effectum hac feliciter deducendi, facilis \& certa ostenditur. In: J. A. COMENII | OPERA | DIDACTICA | OMNIA. | Variis hucusqve occasionibus scripta, | diversisqve locis edita: nunc autem non | tantum in unum, ut simul sint, collecta, sed |

\& ultimo conatu in Systema unum me|chanice constructum, re|dacta. Amsterdam: de Geer. Reprographischer Nachdruck in 3 Bdn. Hrsg. von der tschechoslowakischen Akademie der Wissenschaften. Bd. 1. Prag: Kosmas.

Eggers, Hans (1986): Deutsche Sprachgeschichte. 2 Bde. Reinbek: Rowohlt.

Eisenberg, Peter (2012): Das Fremdwort im Deutschen. 2., überarb. Aufl. Berlin, New York: De Gruyter.

Gardt, Andreas (1997): Das Fremdwort in der Sicht der Grammatiker und Sprachtheoretiker des 17. und 18. Jahrhunderts. Eine lexikographische Darstellung. In: Zeitschrift für deutsche Philologie 116, 388-412.

Gardt, Andreas (2001): Das Fremde und das Eigene. Versuch einer Systematik des Fremdwortbegriffs in der deutschen Sprachgeschichte. In: Gerhard Stickel (Hrsg.), Neues und Fremdes im deutschen Wortschatz. Aktueller lexikalischer Wandel Jahrbuch des Instituts für Deutsche Sprache 2000). Berlin, New York: De Gruyter, 30-58.

Gardt, Andreas et al. (1991): Sprachkonzeptionen in Barock und Aufklärung: Ein Vorschlag für ihre Beschreibung. In: Zeitschrift für Phonetik, Sprachwissenschaft und Kommunikationsforschung 44 (1), 17-33.

Gedike, Friedrich (1779): Gedanken | über Purismus und Sprachbereicherung | von | Friedrich Gedike. | Ut silvae foliis mutantur in annos; | Prima cadunt: ita verborum interit aetas, | Et juvenum ritu florent modo nata vigentque. | HORAT. In: Deutsches Museum 1779 (2), 385-416.

Glaffey, Adam Friedrich (1747): D. Adam Friedr. Glaffeys, | Kön. Pohln. und Chur=Säs. Hof= und Justitien=Raths, | wie auch geheimden Archivarii, | Anleitung | zu einer welt=üblichen Teutschen | Schreib=Art, | Worinnen die | Grund=Lehren | zu dem | in Welt=Händeln gebräuchlichsten | Stylo enthalten sind, | Und | so wohl Stückweise mit Exempeln erläu|tert, als auch am Ende mit gantzen ausgearbei|teten Proben bestärcket werden. 3. und verm. Aufl. Leipzig: Schuster. 
Gottsched, Johann Christoph (1742/1973): Versuch einer critischen Dichtkunst. Erster allgemeiner Theil. In: Ausgewählte Werke. Bd. 6, Teil 1. Hrsg. von Joachim Birke \& Brigitte Birke (Ausgaben deutscher Literatur des XV. bis XVIII. Jahrhunderts). Berlin, New York: De Gruyter. [Originaltitelblatt: Versuch | einer | Critischen Dichtkunst | Durchgehends | mit den Exempeln unsrer besten Dichter erläutert. | Anstatt | einer Einleitung ist Horazens Dichtkunst | übersetzt, | und mit Anmerkungen erläutert, | von | Johann Christoph Gottscheden, | der Weltweish. u. Dichtk. öffentl. Lehrer zu Leipzig. | Dritte und vermehrte Auflage, mit allergnädigster Freyheit. Leipzig 1742.]

Härle, Gerhard (1996): Reinheit der Sprache, des Herzens und des Lebens. Zur Wirkungsgeschichte des rhetorischen Begriffs, puritas' in Deutschland von der Reformationszeit bis zur Aufklärung (Rhetorik-Forschungen 11). Tübingen: Niemeyer. Hallbauer, Friedrich Andreas (1725/1974): M. Friedrich Andreas Hallbauers | Der Hochlöbl. Philosophischen Facultät zu Jena | ADIVNCTI | Anweisung | Zur | Verbesserten | Teutschen Oratorie | Nebst einer Vorrede | von | Den Mängeln | Der Schul=Oratorie. | Mit Kön. Pohlnis. und Churfürstl. Sächsis. gnädigsten PRIVILEGIO. Jena: Hartung. Nachdruck Kronberg i.Ts.: Scriptor.

Hallbauer, Friedrich Andreas (1736/1974): Friedrich Andreas Hallbauers | ELOQVENT. ET POES. PROF. PVBL. ORD. | Anleitung | Zur | Politischen | Beredsamkeit | Wie solche | Bey weltlichen Händeln | In | Lateinisch= und Teutscher Sprache | üblich. Jena, Leipzig: Cröker. Nachdruck Kronberg i.Ts.: Scriptor.

Harsdörffer, Georg Philipp (1645/1969): Frauenzimmer Gesprächspiele. Hrsg. von Irmgard Böttcher. 5. Teil. Reprographischer Nachdruck (Deutsche Neudrucke. Reihe: Barock. Hrsg. von Erich Trunz. 17). Tübingen: Niemeyer. [Originaltitelblatt: GESPRECHSPIELE | Fünfter Theil; | In welchem | Vnterschiedliche / in Teutscher Sprache niebekante | Erfindungen / | Tugendliebenden Gesellschaften auszuüben / Vorgestellet worden: | Benebens einer Zugabe / überschrieben | Die Reutkunst / | Durch einen Mitgenossen der hochlöblichen | FRUCHTBRIN-GENDEN GESELLSCHAFT. Nürnberg: Endter.]

Haßler, Gerda (1984): Sprachtheorien der Aufklärung. Zur Rolle der Sprache im Erkenntnisprozeß (Abhandlungen der Sächsischen Akademie der Wissenschaften, Philologisch-historische Klasse 68, 1). Berlin: Akademie.

Hattenhauer, Hans (1997): Zur Geschichte der deutschen Rechts- und Gesetzessprache. Göttingen: Vandenhoeck \& Ruprecht.

Helwig, Christoph \& Joachim Jung (1614/1615/1893): Artickel / | Auff welchen fürnehmlich die Rati|chianische Lehr Kunst beruhet. [Entstanden um 1614/1615.] In: Ratichianische Schriften II. Mit einer Einleitung und Anmerkungen hrsg. von Dr. Paul Stötzner, Gymnasialoberlehrer in Zwickau. Leipzig: Richter, 11-25.

Hemmer, Jakob (1775): Jakob Hemmers, | kuhrpfälzischen Hofkapellanes, Stiftsherrn zu | Heinsberg, Aufsehers der kuhrfürstlichen Kunstkammer | der Naturlehre, der Mannheimer Gesellschaft der Wissen|schaften, wie auch der landwirtschaftlichen Gesell|schaft zu Lautern Mitgliedes, | Deutsche | Sprachlehre, | zum Gebrauche | der | kuhrpfälzischen Lande. Mannheim: Akad.

Henkel, Nikolaus (2004): Lateinisch/Deutsch. In: Werner Besch et al. (Hrsg.), Sprachgeschichte. Ein Handbuch zur Geschichte der deutschen Sprache und ihrer Erforschung (Handbücher zur Sprach- und Kommunikationswissenschaft 2). Bd. 3. 2. Aufl. Berlin, New York: De Gruyter, 3171-3182. 
Herder, Johann Gottfried (1877): Über die neuere Deutsche Litteratur. Eine Beilage zu den Briefen, die neueste Litteratur betreffend. In: Herders Sämmtliche Werke. Hrsg. von Bernhard Suphan. Bd. 1. Berlin: Weidmann.

Hille, Carl Gustav von (1647/1970): Der Teutsche Palmbaum: | Das ist / | Lobschrift | Von der Hochlöblichen / Fruchtbringenden Gesellschaft | Anfang / Satzungen / Vorhaben / Namen / Sprüchen / | Gemählen / Schriften und unverwelklichem Tugendruhm. | Allen Liebhabern der Teutschen Sprache zu dienlicher | Nachrichtung verfasset / durch den | Vnverdrossenen | Diener derselben. Nürnberg: Endter. Reprographischer Nachdruck in: Die Fruchtbringende Gesellschaft. Quellen und Dokumente in vier Bänden. Hrsg. von Martin Bircher. Bd. 2. München: Kösel.

Jones, William Jervis (Hrsg.) (1995): Sprachhelden und Sprachverderber. Dokumente zur Erforschung des Fremdwortpurismus im Deutschen (1478-1750) (Studia Linguistica Germanica 38). Berlin, New York: De Gruyter.

Kirkness, Alan (1975): Zur Sprachreinigung im Deutschen 1789-1871. Eine historische Dokumentation (Forschungsberichte des Instituts für Deutsche Sprache Mannheim 26). 2 Teile. Tübingen: Narr.

Kirkness, Alan (1985): Sprachreinheit und Sprachreinigung in der Spätaufklärung. Die Fremdwortfrage von Adelung bis Campe. In: Dieter Kimpel (Hrsg.), Mehrsprachigkeit in der deutschen Aufklärung. Berlin: Meiner, 85-104.

Kirkness, Alan (1991): Neuhochdeutsch und Neulatein - eine Begegnung mit dem ,Fremden‘? In: Begegnung mit dem ,Fremden'. Grenzen - Traditionen - Vergleiche. Akten des VII. Internationalen Germanisten-Kongresses Tokyo 1990. Hrsg. von Eijiro Iwasaki. Bd. 4. München: ludicium, 332-340.

Kirkness, Alan \& Horst Haider Munske (Hrsg.) (1996): Eurolatein. Das griechische und lateinische Erbe in den europäischen Sprachen (Reihe Germanistische Linguistik 169). Tübingen: Niemeyer.

Kirkness, Alan (1998): Das Phänomen des Purismus in der Geschichte des Deutschen. In: Werner Besch et al. (Hrsg.), Sprachgeschichte. Ein Handbuch zur Geschichte der deutschen Sprache und ihrer Erforschung (Handbücher zur Sprach- und Kommunikationswissenschaft 2.1). Bd. 1. 2. Aufl. Berlin, New York: De Gruyter, 407-416. Klaj, Johann (1645/1965): Lobrede der Teutschen Poeterey / | Abgefasset | und | in Nürnberg | Einer Hochansehnlich=Volkreichen Versamlung | vorgetragen | Durch | Johann Klajus. Nürnberg: Endter. In: Johann Klaj. Redeoratorien und „Lobrede der Teutschen Poeterey“. Hrsg. von Conrad Wiedemann. Tübingen: Niemeyer, [377]-[416].

Klein, Wolf Peter (2011a): Deutsch statt Latein! Zur Entwicklung der Wissenschaftssprache in der frühen Neuzeit. In: Wieland Eins, Helmut Glück \& Sabine Pretscher (Hrsg.), Wissen schaffen - Wissen kommunizieren. Wissenschaftssprachen in Geschichte und Gegenwart (Fremdsprachen in Geschichte und Gegenwart 8). Wiesbaden: Harrassowitz, 35-47.

Klein, Wolf Peter (2011b): Die deutsche Sprache in der Gelehrsamkeit der frühen Neuzeit. Von der lingua barbarica zur HaubtSprache. In: Herberg Jaumann (Hrsg.), Diskurse der Gelehrtenkultur in der Frühen Neuzeit. Ein Handbuch. Berlin, New York: De Gruyter, 465-516.

Klopstock, Friedrich Gottlieb (1774/1975): Die deutsche Gelehrtenrepublik. In: Werke und Briefe. Historisch-kritische Ausgabe. Abt. Werke, Bd. VII/1. Hrsg. von Rose-Maria Hurlebusch. Berlin, New York: De Gruyter.

Leibniz, Gottfried Wilhelm (1682/1846/1907): Ermahnung an die Teutsche, ihren Verstand und Sprache besser zu üben, samt beigefügtem Vorschlag einer Teutsch gesinnten 
Gesellschaft. [Entstanden um 1682, erstmals veröffentlicht 1846.] In: Paul Pietsch (Hrsg.), Leibniz und die deutsche Sprache (Zeitschrift des Allgemeinen Deutschen Sprachvereins. Wissenschaftl. Beihefte 4 (29)), 290-312.

Leibniz, Gottfried Wilhelm (1697/1717/1908): Unvorgreiffliche Gedancken, betreffend die Ausübung und Verbesserung der Teutschen Sprache. [Entstanden um 1697, veröffentlicht 1717.] In: Paul Pietsch (Hrsg.), Leibniz und die deutsche Sprache (Zeitschrift des Allgemeinen Deutschen Sprachvereins. Wissenschaftl. Beihefte 4 (30)), 313-356. Leibniz, Gottfried Wilhelm (1697/1916): Eine deutschliebende Genossenschaft.

[Entstanden möglicherw. um 1671, vielleicht auch um 1697.] In: Deutsche Schriften.

Bd. 1: Muttersprache und völkische Gesinnung. Hrsg. von Walther Schmied-Kowarzik. Leipzig: Meiner, 55-59.

Litzel, Georg (= Megalissus) (1730): Der Undeutsche | Catholik | Oder | Historischer Bericht | von der allzu grossen Nachläßigkeit der | Römisch=Catholischen, insonderheit unter der | Clerisey der Jesuiten, | In Verbesserung der deutschen | Sprache und Poesie. | Wobey | Die Ursachen solcher Nachläßigkeit an|gezeiget, die eifrige Bemühungen und Verdien| ste der Protestanten zur Nachfolge vorgeleget, | und sichere Mittel zu einer | allgemeinen Sprach=Verbesserung | vorgeschlagen werden | Durch | MEGALISSUS. Jena, Leipzig: Ritter.

Löffler, Heinrich (1991): Deutsch und Latein. Eine tausendjährige Geschichte der Annäherung und der Entfremdung. In: Begegnung mit dem ,Fremden: Grenzen - Traditionen Vergleiche. Akten des VII. Internationalen Germanisten-Kongresses Tokyo 1990. Hrsg. von Eijiro Iwasaki. Bd. 3. München: ludicium, 82-88.

Meier, Georg Friedrich (1763): Georg Friedrich Meiers, | öffentlichen ordentlichen Lehrers der Weltweisheit, | und der Königlichen Akademie der Wissenschaften | in Berlin Mitgliedes, | Betrachtung | über | die Natur | der | gelehrten Sprache. Halle: Hemmerde.

Michaelis, Johann David (1760): Beantwortung der Frage | von dem | Einfluß der Meinungen | in die Sprache | und der Sprache in die Meinungen; | welche den, | von der Königlichen Academie der Wissenschaften | für das Jahr 1759, gesetzten Preis | erhalten hat. | Von | Hrn. Johann David Michaelis, | öffentlichen Lehrer der Weltweisheit in Göttingen und der Königl. | Grosbrittannischen Gesellschaft der Wissenschaften Secretaire. In: Dissertation qui a remporté le Prix proposé par l'Academie Royale des Sciences et Belles Lettres de Prusse, sur l'Influence réciproque du Langage sur les Opinions, et des Opinions sur le Langage. Avec les Pieces qui ont concouru. Berlin: Haude et Spener, 1-84.

Müller, Carl Gotthelf (1754): Daß die | teutschen Gesellschaften | zu unsern Zeiten | nicht allein | den schönen sondern auch den höhern | Wissenschaften | gewidmet seyn sollen | bey | einer ausserordentlichen feyerlichen | Versammlung der Gesellschaft | erwiesen | von | dem Senior derselben. In: Ders. (Hrsg.), Schriften / der Teutschen Gesellschaft / zu Jena I aus den / schönen Wissenschaften / herausgegeben / von / Carl Gotthelf Müller / der Beredsamkeit und Dichtkunst ordentlichem öffentlichen Leh=/ rer, und der Gesellschaft Senior. / auf das Jahr 1753. Jena: Cröker, 1-16.

Parnassus Boicus (1726): PARNASSUS | BOICUS, | Oder | Neu=eröffneter | Musen=Berg /

| Worauf verschidene | Denck= vnd Leßwürdigkeiten | auß der gelehrten Welt / | zumahlen aber | Auß denen Landen zu Bayrn | abgehandlet werden. | Neunzehende | Unterredung. | Mit Erlaubnuß der Oberen. München: Straub.

Polenz, Peter von (1994): Deutsche Sprachgeschichte vom Spätmittelalter bis zur Gegenwart (de Gruyter Studienbuch). Bd. II: 17. und 18. Jahrhundert. Berlin, New York: De Gruyter. 
Pörksen, Uwe (1986): Deutsche Naturwissenschaftssprachen. Historische und kritische Studien (Forum für Fachsprachen-Forschung 2). Tübingen: Narr.

Richter, Johann Gottfried (1784): Johann Gottfried Richters | kritische | Anmerkungen | zu | des Herrn Rath Adelung | deutscher Sprachlehre für die Schulen | in den Königl. Preuß. Landen. Königsberg: Dengel.

Ricken, Ulrich (1989): Leibniz, Wolff und einige sprachtheoretische Entwicklungen in der deutschen Aufklärung. Berlin: Akademie.

Ricken, Ulrich (1990): Sprachtheorie und Weltanschauung in der europäischen Aufklärung. Zur Geschichte der Sprachtheorien des 18. Jahrhunderts und ihrer europäischen Rezeption nach der Französischen Revolution (Sprache und Gesellschaft 21). Berlin: Akademie.

Rivinus, Andreas (1653): Mancherley fürnehmer | Leute JUDICIA | Vrtheil / vnd Meynungen | Vber | Die erste Sprachen=Thür / so | von den Patribus Ibernis in Spanien | erfunden / vnnd Anfangs | gemacht wor|den. | Meistentheils aus dem Latein / verteutschet / / Vnd denjenigen / welche sel|bige anfechten / vnd vnterzudrü|cken gemeint sind / an stadt einer | Vertheidigung / entge|gen gesetzt. Leipzig.

Roelcke, Thorsten (1995): Periodisierung der deutschen Sprachgeschichte. Analysen und Tabellen (Studia Linguistica Germanica 40). Berlin, New York: De Gruyter.

Roelcke, Thorsten (1998): Die Periodisierung der deutschen Sprachgeschichte. In: Werner Besch et al. (Hrsg.), Sprachgeschichte. Ein Handbuch zur Geschichte der deutschen Sprache und ihrer Erforschung (Handbücher zur Sprach- und Kommunikationswissenschaft 2.1). Bd. 1. Berlin, New York: De Gruyter, 798-815.

Roelcke, Thorsten (2001): Einfalt statt Vielfalt? Deutsche und internationale Wissenschaftssprachen im Zeitalter des Rationalismus und in der Gegenwart. In: Jahrbuch der ungarischen Germanistik 2001, 179-192.

Roelcke, Thorsten (2014a): Latein, Griechisch, Hebräisch. Studien und Dokumentationen zur deutschen Sprachreflexion in Barock und Aufklärung (Studia Linguistica Germanica 119). Berlin, Boston: De Gruyter.

Roelcke, Thorsten (2014b): Französisch in Barock und Aufklärung. Studien zum Sprachdenken im Deutschland des 17. und 18. Jahrhunderts (Analecta Romanica 82). Frankfurt a.M.: Klostermann.

Roelcke, Thorsten (2015a): Deutsche Wissenschaftssprache und internationale lingua franca in Aufklärung und Gegenwart. In: Michael Szurawitzki et al. (Hrsg.), Wissenschaftssprache Deutsch - international, interdisziplinär, interkulturell. Tübingen: Narr Francke Attempo, 95-112.

Roelcke, Thorsten (2015b): Einzelsprachen im Werturteil deutscher Sprachdenker des Barock und der Aufklärung. Teil 1: Positive Beurteilungen. In: Zeitschrift für deutsche Philologie 134, 431-467.

Roelcke, Thorsten (2016): Einzelsprachen im Werturteil deutscher Sprachdenker des Barock und der Aufklärung. Teil 2: Negative Beurteilungen. In: Zeitschrift für deutsche Philologie 135, 89-109.

Roelcke, Thorsten (2018): Von der wissenschaftlichen Eignung der deutschen Sprache. In: Michael Prinz \& Jürgen Schiewe (Hrsg.), Vernakuläre Wissenschaftskommunikation.

Beiträge zur Entstehung und Frühgeschichte der modernen deutschen Wissenschaftssprachen. Berlin, Boston: De Gruyter, 361-374.

Schiewe, Jürgen (1996): Sprachenwechsel - Funktionswandel - Austausch der Denkstile. Die Universität Freiburg zwischen Latein und Deutsch (Reihe Germanistische Linguistik 167). Tübingen: Niemeyer. 
Schottelius, Justus Georg (1651): Justi Georgii Schotteli J. V. D. | Teutsche | SprachKunst / | Vielfaltig vermehret und ver|bessert / darin von allen Eigenschaften | der so wortreichen und prächtigen | Teutschen Hauptsprache aus|führlich und gründlich gehan|delt wird. | Zum anderen mahle heraus gegeben. Braunschweig: Zilliger.

Spee, Friedrich (1649/1967): Trutznachtigall. Mit Einleitung und kritischem Apparat hrsg. von Gustave Otto Arlt (Neudrucke deutscher Literaturwerke des XVI. und XVII. Jahrhunderts, begr. von Wilhelm Braune, hrsg. von Ernst Beutler, 292-301). Halle/Saale: Max Niemeyer. [Originaltitelblatt: Trvtz | Nachtigal, | oder | Geistlichs=Poetisch | Lvst=Vvaldlein, | Deßgleichen noch nie zuvor in Teut|scher sprach gesehen. | Durch | Den Ehrw.: P. Fridericvm Spee, | Priestern der Gesellschaft | Jesv. | Jetzo / nach vieler wunsch vnd langem | anhalten / zum erstenmahl in Truck | verfertiget. | Cum Facultate \& approbatione superiorum. Köln: Friessem.]

Stieler, Kaspar (1691/1968): Der | Teutschen Sprache | Stammbaum und Fortwachs | oder | Teutscher | Sprachschatz / | Worinnen | alle und iede teutsche Wurzeln oder Stammwörter / | so viel deren annoch bekant und ietzo im Gebrauch seyn / | nebst ihrer Ankunft / abgeleiteten / duppelungen / und vornemsten | Redarten / mit guter lateinischen Tolmetschung und | kunstgegründeten Anmerkungen befindlich. | Samt einer | Hochteutschen Letterkunst / Nachschuß | und teutschem Register. | So Lehrenden als Lernenden / zu beider Sprachen | Kundigkeit / nötig und nützlich / | durch unermüdeten Fleiß in vielen Jahren gesamlet | von dem | Spaten. Nürnberg: Hofmann. Nachdruck mit einem Nachwort von Stefan Sonderegger. 3 Teile. München: Kösel.

Thieme, Karl Traugott (1789): Ueber die Mittel, bei der Jugend das latei=| nische Sprachstudium zu befördern, von | Herrn Rector Thieme in Merseburg. In: Braunschweigisches | Journal | philosophischen, philologischen und | pädagogischen Inhalts, | Herausgegeben | von E. Chr. Trapp, Joh. Stuve, | Conr. Heusinger, und J. Heinr. Campe. | Erster Band 1789. | Sechstes Stück. Junius 1789. 0.0. 154-218.

Thomasius, Christian (1713): Herrn Christian Thomasens | Sr. Königl. Majestät in Preussen geheimden Raths/ | der Friederich Universität Directoris, Professoris Primarii | und der Juristen Facultaet Decani [etc.]. | Höchstnöthige | CAUTELEN | Welche ein | STUDIOSUS JURIS, | Der sich zu Erlernung | Der | Rechts=Gelahrheit | Auff eine kluge und geschickte Weise vorberei= | ten will/ zu beobachten hat. | Nebst einem dreyfachen und voll=| kommenen Register. | Mit Königlichen Polnischen und Churfürstlichen Säch=| sischen allergnädigsten Privilegio. Halle: Hemmerde.

Weise, Christian (1681/1974): Christian Weisens | Politischer | Redner / Das ist / Kurtze und eigentliche Nachricht / | wie ein sorgfältiger Hofemeister seine Un|tergebene zu der Wolredenheit anführen | sol / damit selbige lernen | 1. Auf was vor ein Fund. eine Schul=Rede gesetzet ist / 2 . Worinn die Complimenten bestehen; 3 . Was bürgerliche Reden sind; | 4. Was bey hohen Personen sonderlich zu Hofe vor | Gelegenheit zu reden vorfällt. | Alles mit gnugsamen Regeln / anständi|gen Exempeln und endlich mit einem nützlichen | Register ausgefertiget / | Auch bey dieser 3ten Edition in vielen | verbessert. | Mit Churfl. Sächs. Privilegio. Leipzig: Ritzsch. Faksimiledruck Kronberg i.Ts.: Scriptor. Wolff, Christian (1733/1973): Christian Wolffens / | Königl. Schwedis. Hochfürstl. Hessis. Hof=Rath | und Prof. Math. \& Phil. prim. zu Marburg, wie auch | Prof.honor. zu St. Petersburg / der Königl. Groß=| Brittannis. und Königl. Preußis. Socität der | Wissenschaften Mitgliedes | Ausführliche | Nachricht | von | seinen eigenen | Schrifften, | die er | in deutscher Sprache | von den | verschiedenen Theilen | der | Welt=Weißheit | heraus gegeben / | auf Verlangen ans Licht gestellet. | Die andere Auflage, hin und 
wieder vermehret. Frankfurt: Andreäische Buchhandlung. Nachdruck in: Gesammelte Werke. 1. Abt.: Deutsche Schriften. Bd. 9. Hrsg. von Hans Werner Arndt. Hildesheim, New York: Olms.

Zesen, Philipp von (1651/1974): Filip Zesens Rosen=mänd. In: Sämtliche Werke. Bd. 11: Spraach-Übung [u. a.]. Bearbeitet von Ulrich Maché. Berlin, New York: De Gruyter. [Originaltitelblatt: Filip Zesens | Rosen=mänd: | das ist | in ein und dreissig gesprächen | Eröfnete Wunder|schacht | zum unerschätzlichen | Steine der Weisen: | Darinnen unter andern ge|wiesen wird / wie das lautere gold | und der unaussprächliche schatz der | Hochdeutschen sprache / unsichtbarlich / I durch den trieb der Natur / von der Zun|gen; sichtbarlich aber durch den / trieb der kunst / aus der fe|der / und beiderseits / | jenes den ohren / | dieses den augen / | vernähmlich / | so wunderbahrerweise und so | reichlich entsprüßet. Hamburg: Pape.] 